\title{
Ilang Katagang Filipino bilang Bukal ng Pamimilosopiya
}

\section{Noel L. Clemente}

\begin{abstract}
Despite Roque J. Ferriols's initiative of teaching philosophy in the Filipino vernacular, many philosophy teachers in the Philippines still use primarily European thinkers, albeit translating their ideas into Filipino. Emerita S. Quito questions the suitability of the Filipino language for philosophizing: can we philosophize in Filipino without merely translating Western ideas? In this paper, I want to dispel Quito's doubts by examining Filipino words that are rich in philosophical meaning, which, I argue, are not merely translations of European ideas. I focus my discussion on key terms in teaching Philosophy of the Human Person: how 'pagninilay' describes what philosophizing is, how 'pagkakataon' captures the historicity of the human person, and how 'tao po' illustrates what philosophy of the human person is about, within a particularly Filipino experience. Through these Filipino terms, I hope to show that our language is indeed rich in philosophical insight.
\end{abstract}

Keywords: philosophizing in Filipino, pagninilay, pagkakataon, philosophy of the human person

\section{Pambungad}

K

araniwang mga Kanluraning pilosopo at teksto ang ginagamit sa pagtuturo ng pilosopiya sa Filipinas-Socrates, Plato, Rene

Heidegger, Gabriel Marcel, at marami pang iba. Hindi naman ito nakagugulat sapagkat ang Europa ang isa sa pinakamatanda at pinakamatatag na kanlungan ng pilosopiya sa mundo. Dahil dito, palasak din ang paggamit ng Ingles bilang wika sa pag-aaral ng pilosopiya-pati na rin ng ibang asignatura - sa mga silid-aralan. Napuna ni Roque J. Ferriols ang kabalintunaan ng ganitong kalakaran: bukod sa pagpapabása sa mga magaaral ng mga artikulong nása banyagang wika, pinag-iisip din sila sa isang

(C) 2018 Noel L. Clemente

https://www.kritike.org/journal/issue 23/clemente december2018.pdf

ISSN 1908-7330

$(c c)$ BY-NC-ND 


\section{ILANG KATAGANG FILIPINO}

wikang hindi kinasanayan ng kanilang diwa. ${ }^{1}$ Ito ang nagtulak sa kaniyang isulong ang pagtuturo ng pilosopiya sa wikang Filipino noong $1969 .{ }^{2}$

Sa pambungad sa isang Festschrift para kay Ferriols, inilalagom nina Nemesio Que at Agustin Rodriguez ang kahalagahan ng pagtuturo ng pilosopiya at pag-iisip sa katutubong wika:

Kung magtuturo ka ng buháy na pag-iisip sa mga magaaral, kailangan silang mapag-isip sa kanilang buháy na wika. Ibig sabihin, hindi ang wikang pormal na hitik sa naisaulong pormula, kundi ang wikang ginagamit nila sa pagdanas ng katalagahan at pamumuhay sa kanilang mundo. Samakatwid, may pangangailangang mamilosopiya sa wikang Filipino. ${ }^{3}$

Nagpapatúloy ang ganitong tradisyon ng pamimilosopiya ${ }^{4}$ at pagtuturo ng pilosopiya sa wikang Filipino ${ }^{5}$ na pinasiklab ni Ferriols. Ngunit dahil na rin sa kasanayan ng mga Filipino sa pilosopiyang Europeo, hindi maiiwasan ang paghahambing ng pamimilosopiya ni Ferriols sa mga ideya ng mga Europeo. Halimbawa, may mga kumukuwestiyon kung ang meron $^{6}$ ba na tinatalakay

\footnotetext{
${ }^{1}$ Nemesio S. Que and Agustin Martin G. Rodriguez, eds., Introduction to Pagdiriwang sa Meron: A Festival of Thinking Celebrating Fr. Roque J. Ferriols, S.J. (Quezon City: Ateneo de Manila University, 1997), vi.

${ }^{2}$ Para sa kasaysayan at konteksto ng pagpapasiya ni Ferriols na magturo ng pilosopiya sa Filipino, tingnan ang Franz Giuseppe F. Cortez, "The Linguistic Turn as a Political Act: Another Look at the Thoughts of Roque Ferriols," Kritike: An Online Journal of Philosophy, 8:1 (June 2014), 51-55, pati na rin ang kinalap ni Glenda C. Oris na panayam sa "Ferriols on Ferriols and More," Perspectives in the Arts and Humanities Asia 5:1 (2015), 121-125.

${ }^{3}$ Salin ng may-akda sa "If one were to teach living thought to students, they need to be made to speak in their living language. That is to say not the formal language of memorized formulas and petrified discourse, but the language with which they experience their world and live their reality. Thus, the necessity to allow philosophy to speak in Filipino," mula sa Rodriguez and Que, Introduction, vii.

${ }^{4}$ Nananatiling bukal ng pananaliksik ang pilosopiya ng "meron" ni Ferriols, pati na rin ang kaniyang pamamaraan ng pamimilosopiya sa katutubong wika. Ilan sa mga kontemporanyong artikulo ang mga sumusunod: Roy Allan B. Tolentino, "Biyaya ang Meron, Biyaya ng Meron: Ang Pag-iisip nina Ferriols, Marion, at San Agustin," Perspectives in the Arts and Humanities Asia 5:1 (2015), 91-107; Albert M. Lagliva, "Ang Paraan at mga Elemento ng Pagtuturo ni Roque J. Ferriols, SJ, tungo sa Matinong Pag-Uunawa," Perspectives in the Arts and Humanities Asia 5:1 (2015), 41-53; at Wilhelm P.J. Strebel, "Pitong Sulyap sa Pilosopiya ng Wika ni Ferriols," Kritike: An Online Journal of Philosophy, 12:1 (June 2018), 39-55.

${ }^{5}$ Sa kasalukuyan, halos kalahati ng mga kurso sa pilosopiya sa Pamantasang Ateneo de Manila ay itinuturo sa wikang Filipino. Ilan sa kanila sina Remmon E. Barbaza, Eduardo Jose E. Calasanz, Preciosa A. De Joya, Albert M. Lagliva, Michael Ner E. Mariano, Mark Oliver G. Pasco, Jovino G. Miroy, Agustin Martin G. Rodriguez, Wilhelm P.J. Strebel, at Venusto F. Suarez.

${ }^{6}$ Matatagpuan ang ekstensibong pamimilosopiya ni Ferriols ukol sa meron sa kaniyang Pambungad sa Metapisika (Quezon City: Ateneo de Manila University Press, 2014).
}

(C) 2018 Noel L. Clemente

https://www.kritike.org/journal/issue 23/clemente december2018.pdf

ISSN 1908-7330

(c) BY-NC-ND 
ni Ferriols ay katulad ng Sein ni Heidegger. ${ }^{7}$ Lumilitaw rito ang suliranin ng potensiyal ng wikang Filipino sa pilosopiya: káya ba nating gamitin ang sariling wika sa pamimilosopiya nang hindi lámang isinasalin ang mga ideya ng Europeo?

Sa palagay ni Emerita S. Quito, hindi. Isa sa mga dahilan kung bakit naniniwala siyang walang maituturing na pilosopiyang Filipino ${ }^{8}$ ay dahil hindi kondusente ang wikang Filipino sa pagtalakay ng mga abstraktong paksa ng pilosopiya. Isinusulat niya: "Maging ang wika natin ay hindi bagay sa pilosopiya. Walang katumbas sa Filipino ang mga salitâng being, essence, existence, becoming, actuality, transcendence." ${ }^{9}$

Tinatawag ito ni Albert E. Alejo na isa sa mga "tiyanak ng kalooban," mga mapanuksong elemento na sinusubok linlangin at iligaw ang mga manlalakbay sa pag-iisip. ${ }^{10}$ Dahil sa ganitong pangamba sa kakayahan ng sariling wikang maipahayag ang mga pilosopikong dalumat, may mga nagaalinlangan na gamitin ang Filipino sa pamimilosopiya. Ang kadalasang paratang: Filipino man ang wikang ginagamit, mga Europeong ideya pa rin ang pinag-iisipan, at isinasalin lámang ang mga ito sa ating wika. At kung hindi man mahanapan ng katumbas na salita sa Filipino, ninanakaw na lang ang mga salitang pilosopiko: Angst, Sein, differánce, atbp.

Sa papel na ito, nais kong tuligsain ang haka ni Quito; gusto kong ipakita na may pangako ang wikang Filipino para sa pamimilosopiya sa kabila ng mga di maisasaling kataga. ${ }^{11}$ Ani Ferriols, "may natatanging potensiyal ang bawat

7 Halimbawa, para kay Leonardo Mercado, ang ideya ng "meron" ni Ferriols ay maisasalin bilang "being." Taliwas dito, iginigiit ni Mark Joseph Calano na hindi ito salin ng being ng Ingles, Sein ng Aleman, être ng Pranses. Ayon sa kaniyang panayam kay Ferriols, ni hindi pa nabasa nitong hulí ang Being and Time ni Martin Heidegger. Tingnan ang Mark Joseph Calano, "Ang Pilosopiya at si Roque J. Ferriols, SJ," Perspectives in the Arts and the Humanities 5:1 (2015), 15.

${ }^{8}$ Isang kontrobersiyal na paksa noong dekada sitenta ang suliranin ng kung mayroon bang matatawag na "pilosopiyang Filipino," na tinatalakay pa rin hanggang ngayon. Bagaman interesante ang usaping ito, nalilihis na ito sa paksa ng papel ko. Maaaring tingnan ang iba't ibang pananaw sa Roque J. Ferriols, "A Memoir of Six Years," Philippine Studies 22 (1974), 339340, Leonardo N. Mercado, Elements of Filipino Philosophy (Tacloban City: Divine Word University Publications, 1993), 3-4; at Emerita S. Quito, Lectures on Comparative Philosophy (Manila: De La Salle University Press, 1979), 22-29.

${ }^{9}$ Salin ng may-akda sa "Even our langauge does not lend itself to philosophy. There are no equivalents to words like being, essence, existence, becoming, actuality, transcendence," mula sa Quito, Lectures on Comparative Philosophy, 29.

${ }^{10}$ Albert E. Alejo, Tao pô! Tulóy! Isang Landas ng Pag-unawa sa Loob ng Tao (Quezon City: Ateneo de Manila University Office of Research and Publications, 1990), 6.

${ }^{11}$ Hindi ko nais ipahiwatig dito na sinasalungat ni Quito ang proyekto ng paggamit ng wikang Filipino sa pamimilosopiya. Sa katunayan, iginigiit niya ang kahalagahan ng paggamit ng ating wika sa iba't ibang larang, kasama na ang pilosopiya, upang mapaunlad ang wika, sa "Ang Kayamanan ng Wikang Filipino," in A Life of Philosophy: Festschrift in Honor of Emerita S. Quito (Manila: De La Salle University Press, 1990), 604-605. Nais kong bigyang-diin na ang tinutuligsa ko rito ay hindi ang hamak na posibilidad ng pamimilosopiya sa wikang Filipino, 


\section{ILANG KATAGANG FILIPINO}

wika sa pagtingin at pagdama,"12 at dahil dito, may nabibigkas ang ating wika na marahil hindi maaaring bigkasin sa Ingles, Pranses, Aleman, o iba pang wika (katulad din ng alegasyon na hindi mabibigkas sa Filipino ang being, transcendence, etc. na pinag-usapan ng mga Europeo). Ipapakita ko, gamit ang tatlong halimbawa ng mga katagang Filipino, na naglalaman ang ating wika ng mga karanasang bukal ng pilosopikong pagninilay.

Igigiit kong ang mga katagang Filipino na tatalakayin ko sa papel na ito, taliwas sa pangamba ni Quito, ay hindi hamak na pagtatangkang (o pagpipílit) isalin ang mga ideyang Europeo sa sarili nating salita. Hindi ko naman inimbento ang mga katagang ito, maging ang kanilang mga kahulugan; karaniwan nang ginagamit ang mga salitang ito bagaman hindi namamalayan ang kanilang pilosopikong pahiwatig. Sapagkat "taal na paraan ng pamumuhay ang bawat wika," 13 may nilalaman ang mga katagang ito na nagkataon lámang na kahawig - ngunit hindi ganap na katumbas-ng mga pilosopikong ideya ng Europa. Sa katunayan, walang monopolyo ang alinmang wika sa pamimilosopiya; nauna mang mailimbag sa Pranses o sa Aleman ang mga ideya ng mga pilosopong Europeo, hindi ito nangangahulugang walang ganoong katotohanan sa ating wika

Samakatuwid, nais kong ipakita sa papel na ito na nararanasan din nating mga Filipino, sa pamamagitan ng ating wika, ang mga katotohanang naipahayag na sa ibang wika. Kung tutuusin, sinusubukan naman ng pilosopiyang pag-usapan ang mga unibersal na karanasan ng tao sa mundo, ngunit matutunghayan natin sa ating wika ang pagka-partikular nito sa ating danas. Babanggitin ko ang mga ideya ng mga Europeong pilosopo dahil ito marahil ang mas kilalá ng mambabasa (lalo na sa mga nag-aaral ng pilosopiya), ngunit hindi ito nangangahulugan na hinango ko ang kahulugan ng mga katagang Filipino sa mga pilosopong Europeo. ${ }^{14}$ Muli, bagaman may pagkakahawig, hindi sila ganap na magkatumbas.

Hindi sasapat ang maikling papel na ito (o maging isang buong aklat) para suriin ang lahat ng mga salitang may taglay na malalim na pilosopikong danas. Kayâ pagtutuunan ko ng pansin ang tatlong kataga na sa tingin ko'y

kundi ang paggamit ng ating wika nang hindi basta isinasalin ang mga pilosopiya ng mga Europeo.

12 Salin ng may-akda sa "Each language has unrepeatable potentials for seeing and feeling," mula sa Ferriols, "A Memoir of Six Years," 340.

${ }^{13}$ Salin ng may-akda sa "Each language is a way of being alive that is irreducible," mula sa ibid., 340 .

${ }^{14}$ Kahawig ito ng estratehiya ni Leonardo Mercado sa pagtatangkang makausap ang mga mambabasang mas nasanay sa pag-aaral ng pilosopiyang Kanluranin. Ipinapaliwanag niya na itinatapat lang niya ang nililinang niyang pilosopiyang Filipino sa mga kaisipang Kanluranin para mas maunawaan ng mga mambabasa ang kaniyang ipinapaliwanag, ngunit iginigiit niya na hindi ito pagmamaliit sa mga kaisipang Filipino. Tingnan ang Mercado, Elements of Filipino Philosophy, 13.

(C) 2018 Noel L. Clemente

https://www.kritike.org/journal/issue 23/clemente december2018.pdf

ISSN 1908-7330

(cc) BY-NC-ND 
akmang inilalahad ang mga sentral na ideya ng Pilosopiya ng Tao: una, kung ano ang pilosopiya; ikalawa, kung ano ang tao; at ikatlo, kung ano ang pamimilosopiya ukol sa tao. Titingnan ko sa mga susunod na bahagi ang pilosopiya bílang isang pagninilay, ang táong sumasakasaysayan bílang pagkakataon, at ang pamimilosopiya ukol sa tao bílang Tao po! Sa pamamagitan ng tatlong halimbawang ito, umaasa akong mamumulat ang mambabasa sa pagkabukal ng wikang Filipino sa pamimilosopiya.

\section{Pamimilosopiya bilang Pagninilay}

Karaniwang ginagamit ang kurso ng Pilosopiya ng Tao bílang pambungad sa pilosopiya. ${ }^{15}$ Dahil dito, isa sa mga pinakamahahalagang maunawaan ay kung ano nga ba ang pilosopiya, at kung paano ito naiba sa ibang uri ng pag-iisip. Iginigiit ni Ferriols na "Lalong mabuting gawin muna bago pag-usapan kung ano [ang pilosopiya]. Sapagkat ang pilosopiya ay ginagawa."16 Inihahambing niya ang pamimilosopiya sa paglalakad, pamimisikleta, paglalangoy, at pagmamaneho: mga gawaing natututuhan ng tao sa mismong paggawa nito. Sa kabila nito, hindi maiiwasan ng táong maghanap ng mapanghahawakang paunang ideya o konsepto ng kung ano ang pilosopiya. Maaari itong mabasag at maitama kalaunan, gayunpaman likás sa tao na magsimula sa isang konsepto sa pagkatuto.

Kaugnay nito, kadalasang ikinakabit ang ideya ng pagninilay o pagmumuni sa pamimilosopiya. Itinuturing ng mga tao na mapagnilay ang pilosopiya. Ngunit tulad ng pagpapaliwanag ni Gabriel Marcel, kadalasan namang iniuugnay ang pagninilay sa isang 'malalim' na gawain, na tíla ba kailangan mong magkulong sa iyong silid at lumayo sa ibang tao upang makapagnilay nang maayos. ${ }^{17}$ Sa gayon, tinitingnan ang mga pilosopo, o sinumang palánilay, bílang mga "malalalim" na tao na para bang lutáng sa mundo. Nariyan ang kuwento ng sinaunang pilosopong Griyego na si Thales na naglalakad nang nakatingala sa mga bituin at sapagkat hindi nakatingin sa dinaraanan ay nahulog sa balon. Makikita rin ang ganitong karikatura ng mga pilosopo sa sining at panitikan: nariyan si Pilosopo Tasyo ng Noli Me Tangere na inilalarawan bílang may sariling mundo at hindi gaanong

15 Sa kasalukuyang "core curriculum" (mga asignaturang inaaral ng lahat ng estudyante, anuman ang kanilang major) ng kolehiyo sa Pamantasang Ateneo de Manila, ang Pilosopiya ng Tao ang unang kurso sa pilosopiya ng mga mag-aaral. Dahil dito, ang unang paksa sa kursong ito ay pagtatanong sa kung ano nga ba ang pilosopiya.

${ }^{16}$ Ferriols, Pambungad sa Metapisika, ix.

17 Ipinapaliwanag ni Gabriel Marcel sa unang bahagi ng ikalimang kabanata ng kaniyang aklat ang ganitong karaniwang palagay ukol sa pagninilay, at pinapabulaanan niya ito sa pagpapakitang nakaugat ang pagninilay sa búhay. Tingnan ang Gabriel Marcel, Reflection and Mystery, vol. 1 of The Mystery of Being, trans. by G.S. Fraser (London The Harvill Press, 1951), $77-$ 82. Mula rito, tutukuyin ang akda bilang The Mystery of Being.

(c) 2018 Noel L. Clemente

https://www.kritike.org/journal/issue 23/clemente december2018.pdf

ISSN 1908-7330

(cc) BY-NC-ND 


\section{ILANG KATAGANG FILIPINO}

kinakausap ng mga tao, pati na rin ang eskultura ni Auguste Rodin na The Thinker na nagpapakitang ang isang palaisip ay karaniwang mag-isa.

Taliwas sa karaniwang konsepto patungkol sa pilosopiya, may sinasabi ang mismong salitang pagninilay ukol sa kung ano ba talaga ang pamimilosopiya. Maaaring ituring na salitang ugat nito ang silay, ${ }^{18}$ na nangangahulugang pagtingin. Samakatuwid, bago pa man tayo sumisid sa kalaliman ng ating loob, ang pagninilay ay isa munang pagtingin. Ipinapakita ni Marcel sa karanasan ng pagkawala ng kaniyang relo na ang pagninilay ay nagsisimula sa pagbibigay-pansin sa mga bagay na mahalaga sa isang tao. ${ }^{19}$ Pamilyar ang ganitong karanasan: kapag nawawalan ako ng relo, o cellphone, pitaka, susi, o anumang bagay na hindi ko matagpuan sa karaniwang kinalalagyan, aalalahanin ko kung saan ko ito hulíng nakita, hahalughugin ang mga lugar na pinanggalingan ko, hanggang sa matagpuan ko ang nawawala. Nagsisimula ang pagtingin sa ganitong mga bitak sa karanasan: may nawawala, may bago, may kakaiba, may hindi ako napansin dati kayâ nagtataka ako ngayong napansin ko na. Ayon sa mga sinaunang Griyego, ito ang thaumazein o wonder na sinasabing simula ng pamimlosopiya; nagaganap lamang ang pagtataka at pagkamangha kung tumingin muna ako. ${ }^{20}$ Ito rin ang hámon ni Wittgenstein: "Look and see!" para matuklasan kung paano gumagana ang wika. ${ }^{21}$

Ganito rin ang ipinahihiwatig ng salita ng mga sinaunang Griyego sa katotohanan. Para sa kanila ang katotohanan ay aletheia: pagkakawala sa pagkakakubli, samakatuwid, pagpapakita. ${ }^{22}$ Masasaksihan lamang ng isang tao ang nagpapakita kung sisilayan niya ito. Kung hindi matalas ang kaniyang pagtingin, ang kaniyang pagsilay, hindi niya makikita ang nagpapakita. Sang-ayon ito sa isa pang karaniwang konsepto ng pilosopiya bilang paghahanap ng katotohanan: masisimulan ko lang mahanap ang katotohanang nagpapakita (aletheia) kung titingnan ko ito (pagninilay at pamimilosopiya).

Dagdag pa rito, ipinapaliwanag ni Ferriols na kung nais mong makita, kailangang 'ikaw' ang tumingin. ${ }^{23}$ Walang ibang puwedeng

18 Ayon sa UP Diksiyonaryong Filipino, binagong ed., s.v. "nilay", "nilay" ang tinukoy na salitang ugat ng "pagninilay." Ang iminumungkahi kong pag-uugnay ng "nilay" sa "silay" ay sarili kong interpretasyon, na inaasahan kong maaaring pagmulan ng hinuhang pilosopiko.

${ }^{19}$ Marcel, The Mystery of Being, 77-78.

${ }^{20}$ Aristotle, Metaphysics, trans. by W.D. Ross, in The Basic Works of Aristotle, ed. by Richard McKeon. (New York: Random House, 1941), 982b 11-24.

${ }^{21}$ Ludwig Wittgenstein, Philosophical Investigations, rev. 4th ed., ed. by P.M.S. Hacker and Joachim Schulte, trans. by G.E.M. Anscombe, P.M.S. Hacker, and Joachim Schulte (Malden: Wiley-Blackwell, 2009), §§ 66, 72, 93, 578.

22 Pansinin na sinasalamin ng salitang aletheia ang karansan ng mga sinaunang Griyego sa pamimilosopiya, sa parehas na paraan na ipinakikita kong nakikita sa pagninilay o pagsilay ang karanasan ng Filipino sa pamimilosopiya.

${ }^{23}$ Ferriols, Pambungad sa Metapisika, xi-xii.

(c) 2018 Noel L. Clemente

https://www.kritike.org/journal/issue 23/clemente december2018.pdf

ISSN 1908-7330

(cc) BY-NC-ND 
tumingin para sa iyo; maaaring ikuwento ng ibang tao ang nakita nila sa iyo, ngunit kung mananatili ka lang sa pakikinig ng kanilang kuwento, wala kang makikita. Samakatuwid, lubos na personal ang karanasan ng pagtingin. Ang aking pagtingin ay maaaring hindi katulad ng pagtingin ng ibang tao, bagaman magkaparehas kami ng bagay na tinitingnan. Lumilitaw rito ang iginuhit ni Marcel na pagkakaiba ng pagmumuning primarya at sekundarya. ${ }^{24}$ Paliwanag niya, ang pagmumuning primarya ay isang obhetibong pagtanaw sa bagay; nagmumula ang 'obheto' sa ob-jectum na nangangahulugang "pagtatapon sa harap." Samakatuwid inilalagay ko sa harap ko ang bagay na pinagmumunihan, na tíla isang bagay na hiwalay sa akin. Dahil hiwalay ito sa akin, maaari ko itong suriin, kalikutin, at kumpunihin, upang maunawaan nang maayos. Mahalaga rito ang pagkakahiwalay ng nagninilay sa pinagninilayan; may agwat na tumutulot sa pagsusuri. Karaniwang itinuturing na obhetibo ang mga agham at matematika sapagkat nararapat na may paghihiwalay ang mga siyentista at matematiko sa kanilang inaaral. Ang mga suliraning ganito ay maaaring sagutin at pag-isipan ninuman, at inaasahan na magkatulad lamang ang mga sagot na makukuha, sapagkat hindi naman nakasalalay ang suliranin sa umuunawa nito.

Sa kabilâng banda, ang pagmumuning sekundarya naman ay suhetibo; ang 'suheto' ay mula naman sa sub-jectum bilang "pagtatapon sa ilalim." Hindi ganoon kadaling ihiwalay ang nagninilay sa pinagninilayan, dahil bahagi ng pagninilay ang nagninilay. Halimbawa, ang mga opinyon ay inilalarawan bilang suhetibo sapagkat hindi maihihiwalay ang mga ito sa taong pinanggalingan nito; ang magkakaibang tao ay may magkakaibang opinion. Sangkot siya sa mismong pagtingin, at hindi lamang hamak na tagamasid. Ganito ang nais ipahiwatig ni Ferriols sa kaniyang sikát na paanyaya: "Lundagin mo beybe!" 25 Kailangan mong tumingin kung nais mong ikaw ang makakita. Bagaman maaaring pag-aralan ang pilosopiya bilang isang obheto sa pamamagitan ng pagbabasa at pag-uunawa ng lahat ng akda ng mga dakilang pilosopo sa mundo, hindi pa ito pamimilosopiya. Maihahalintulad lamang ito sa pagbabasa ng manual ng paglangoy o pagmamaneho nang hindi pa lumulusong sa tubig o humahawak ng manibela (mga halimbawa ng pagmumuning primarya). Hangga't hindi sarili mismo ang tumitingin - hindi bilang tagamasid kundi bilang kasangkot

\footnotetext{
24 Tinatalakay ni Marcel sa ikalimang kabanata ng The Mystery of Being (pp. 83-93) ang dalawang uri ng pagninilay sa kaniyang pagtingin sa karanasan ng pagsasakatawang-diwa ng tao. Maaari ring tingnan ang salin ng kabanatang ito sa Filipino: "Ang Bulangang 'Heto Ako!'," trans. by Nonna J. Peña, in Magpakatao: Ilang Babasahing Pilosopiko, 2nd ed., ed. by Roque J. Ferriols (Quezon City: Office of Research and Publications, Ateneo de Manila University, 1999), 104-128.

${ }^{25}$ Ferriols, Pambungad sa Metapisika, $\mathrm{x}$.
} 


\section{ILANG KATAGANG FILIPINO}

ng karanasan ${ }^{26}$-hangga't hindi lumulundag ang sarili, hindi pa ganap na matatawag na namimilosopiya ang tao.

Ang hulíng puntong nais kong talakayin ay ang hamon ng pamimilosopiya. Kung ito ay tunay na pagninilay, isang tuwinang pagsilay, bakit naituturing na mahirap at di-pangkaraniwan ang pilosopiya? Kailangan nating maunawaan kung bakit itinuturing higit na mahirap ang isang pang-araw-araw na gawain tulad ng pagtingin.

Kung tutuusin, likás sa mga bata ang pagninilay sapagkat nabibihag pa sila ng pagkamangha sa mga bagay. Lahat ng nakikita nila ay bago, kayâ tanong sila nang tanong. Dumaan ang lahat ng tao sa ganitong yugto. Ngunit sa pagkatuto at pagkasanay sa mga bagay sa mundo, nalimutan na natin ang katutubong pananabik matuto (thaumazein, wonder): dahil nalimutan na nating sumilay. Ika ni Ferriols, nababara na tayo sa awtomatiko. ${ }^{27}$ Nasanay na tayo sa mga konsepto kayâ wala nang pagnanasàng sumilay at tumingin. May tiyak na táyong inaasahan sa mga bagay buhat sa ating mga natutuhan. May pagsasantabi sa mga bagong karanasan dahil "alam ko na iyan."

Ito ang hamon ng pilosopiya, na binigkas na ng maraming pilosopo sa nakaraan-ang panatilihin ang pananabik matuto sa kabila ng mga nakasanayan sa mundo. Hinahamon táyong huwag magsawang tumingin at sumilay, kahit sa mga bagay na natingnan at nasilayan na natin dahil hindi kailanman mauubos ang matututuhan natin ukol sa isang bagay.

Samakatuwid, ang pilosopiya ay isang walang sawa at patúloy na pagninilay: ang parating pagtataka at pagtingin sa talaga.

\section{Ang Pagkasumasakasaysayan ng Tao bílang Pagkakataon}

Nakita natin sa nakaraang yugto kung paanong nilalaman ng salitang pagninilay ang kay raming pilosopikong ideya, lalo na kaugnay sa paglalarawan ng kung ano ang pilosopiya. Sa bahaging ito, ilalarawan naman natin ang pagkasumasakasaysayan ng tao sa pamamagitan ng pagkakataon.

Isa sa mga pinakamahalagang paksa, sa palagay ko, na itinatalakay ng Pilosopiya ng Tao, ay ang relasyon ng tao sa kasaysayan. Ipinaliliwanag ni Ramon C. Reyes na "ang tao ay hugpungan ng mga pangyayari." 28 Naroroon ang isang tao sa kinalalagyan niya ngayon dahil sa mga nangyari sa nakaraan, at ihahatid siya ng mga pangyayari sa kasalukuyan sa kung saan man siya mapupunta sa hinaharap. Samakatuwid, ang tao ay bahagi ng

\footnotetext{
${ }^{26}$ Makikita rito ang sinasabi ni Alejo na ang pilosopiya ay isang pakikisangkot. Tingnan ang Tao pô! Tulóy!, 4-5.

${ }^{27}$ Ferriols, Pambungad sa Metapisika, xi.

${ }^{28}$ Salin ng may-akda sa "Man is the 'cross-point' or point of intersection of certain lines of events," mula sa Ramon Reyes, "Man and Historical Action," in Philosophy of Man: Selected Readings, 3rd ed., ed. by Manuel B. Dy, Jr. (Quezon City: Katha Publishing Co., Inc., 2012), 109.

(C) 2018 Noel L. Clemente

https://www.kritike.org/journal/issue 23/clemente december2018.pdf ISSN 1908-7330

(c) $\mathrm{BY}-\mathrm{NC}-\mathrm{ND}$
} 
kasaysayan, at nakikibahagi rin sa paglikha nito; ang tao ay sumasakasaysayan.

Ang sentral na mensahe ni Reyes: parehas na limitasyon at posibilidad ang kasaysayan ng tao. ${ }^{29}$ Ito ang dalawang mukha ng ating pagkasumasakasaysayan. Sapagkat ako ay hugpungan ng mga pangyayari, limitado ang aking maaaring galawan sa mga nangyari na't hindi ko na mababago. Ngunit saanman ako naroroon, mayroon akong mga posibilidad na maaari kong patunguhan. Ang pagiging limitasyon at posibilidad ng ating kasaysaysan ay nagsasanib sa salitang pagkakataon. Kadalasan itong ginagamit bilang oportunidad, ngunit maaari rin itong gamitin bilang koinsidensiya (tulad ng paggamit sa "nagkataon lang"). Ipaliliwanag ko ngayon ang punto ni Reyes sa pamamagitan nitong dalawang gamit ng pagkakataon.

Naririto táyo sa ating kinatatayuan ngayon dahil nangyari na ang mga nangyari sa nakaraan, at wala táyong magagawa sa mga nangyari na. Sinakop na ng mga Kastila, at ng mga Amerikano ang Filipinas; nanungkulan na ang iba't ibang presidente; nagtagpo ang aking mga magulang; bininyagan akong Katoliko Romano; pinaaral ako sa ganitong paaralan; nakilala ko ang mga táong ito; pinili ko itong partikular na landas noong nakaraang taon - at dahil nagkataon ang lahat ng mga ito, naririto ako sa aking kasalukuyan. Samakatuwid, limitado ang aking kasalukuyan ng mga koinsidensiya ng nakaraan. Ito ang dahilan kung bakit makatwirang isisi sa nakaraan ang anumang kasalukuyang kinalalagyan. Kung hindi ko nasagutan ang aking pagsusulit, maaari kong sisihin ang pagkakataong tinamad akong mag-aral kagabi. Kung naging suwail at rebelde ang aking pag-uugali, maaari kong sisihin ang pagkakataon na mahigpit ang pagpapalaki sa akin ng aking mga magulang. Kung kinasusuklaman ko ang Kanluraning impluwensiya sa aking pag-iisip, maaari kong sisihin ang aksidenteng pagkakatagpo ni Magellan sa Mactan noong 1521, o ang pananakop ng mga Amerikano sa bansa noong simula ng ika-dalawampung siglo.

Ngunit iginigiit ni Reyes na tila hindi makatwiran para sa isang tao na habambuhay na isinisisi na lamang sa nakaraan ang kaniyang kalagayan. Isinalaysay ni Reyes ang dati niyang estudyante na isinisisi sa "katangahan at kawalan ng ambisyon" ng kaniyang ama ang kaniyang kahirapan. Nakilala niya itong estudyante nang nása ikalawang taon ito ng kolehiyo, at magpahanggang ikaapat na taon ay patúloy ang paninisi ng estudyante sa kaniyang ama. ${ }^{30}$ Bagaman may katwiran siya sa ganitong paninisi, hindi na yata katanggap-tanggap kung hanggang maging $30 \mathrm{o} 40$ anyos siya ay sa ama

\footnotetext{
${ }^{29}$ Reyes, “Man and Historical Action," 113-114.

${ }^{30}$ Ibid., 113.
}

(c) 2018 Noel L. Clemente 


\section{ILANG KATAGANG FILIPINO}

pa rin niya isisisi ang kaniyang kalagayan sa búhay. Maihahalintulad ito sa isang laro ng baraha. Kapag nakikipaglaro ka sa iyong mga kaibigan ng baraha, binabalasa (kung walang nandaraya) nang tapat ang mga baraha. Sa unang minuto, pamilyar na eksena ang paninisi sa sinumang nagbalasa kung bakit pangit ang mga barahang nakuha ng isang manlalaro. Nagkataon lang naman na yaon ang mga napunta sa kaniya, at wala na siyang magagawa; limitado ang tsansa niyang manalo sa kalidad ng mga baraha niya. Ngunit hindi yata makatwiran kung lilipas ang isang oras at hindi pa rin siya nakikipaglaro dahil patuloy ang paninisi niya sa kalarong nagbalasa. Kailangang umabot sa punto na tanggapin niya ang mga barahang naibigay sa kaniya at maglaro na lang gamit ng mga ito.

Dito pumapasok ang posibilidad ng kasaysayan na kaakibat ng mga limitasyon nito; sapagkat nagkataon (konsidensiya) ang mga pangyayari sa nakaraan, mayroong pagkakataon (oportunidad) ang tao na kumilos at magpasiya. ${ }^{31}$ Ang nagkataong pangkat ng mga baraha na napunta sa isang manlalaro ang siya ring nagbibigay sa kaniya ng pagkakataong maglaro. Hindi man niya mapipili kung aling mga baraha ang mapupunta sa kaniya, mapipili naman niya kung paano niya lalaruin ang mga ito. Hindi dapat limutin ang pagkakatali ng kaniyang mga posibilidad sa kaniyang limitasyon; mahihirapan man siyang manalo kung pangit ang kaniyang mga baraha, hindi nito ibig sabihing imposible ang manalo. Gayundin, mahihirapang mamuhay nang maayos at matiwasay ang isang táong hindi pinagpalà ng mga biyaya sa búhay, ngunit may káya pa rin siyang gawin sa anumang kakaunti na naibigay sa kaniya. Sa kabilâng banda, hindi rin naman ibig sabihin na kung magaganda ang baraha ay mananalo na agad ang isang manlalaro, tulad ng hindi ibig sabihin na kung marangya at sagana ang pinanggalingan ay madadalian na siyang makipagsapalaran sa búhay. Dito natin makikita na hindi sapat ang suwerte sa laro (at sa búhay); mahalaga rin ang diskarte.

Samakatuwid, bagaman limitado ang ating kasaysayan, hitik din tayo sa posibilidad dahil dito. Sapagkat naririto na ako sa aking kinatatayuan, marami akong maaaring arami akong maaaring tahaking landas. Nagkataong ipinanganak ako sa Filipinas, at isa itong pagkakataong magpaka-Filipino. Maaari kong piliing lisanin ang bansa kalaunan o maaari akong manatili, pero anupaman ang mangyari, mayroon akong pagkakataon. Nagkataong mga Katoliko ang aking mga magulang kayâ ako bininyagang Katoliko, at isa itong pagkakataong magpaka-Katoliko, o maaari ring pagkakataong tumiwalag sa aking relihiyon. Nagkataong naging kaklase ko

\footnotetext{
${ }^{31}$ Pinuna ni Donald Jay Bertulfo na mas palasak ang gamit ng mga Filipino sa salitang "pagkakataon" bilang oportunidad kaysa koinsidensiya. Maaaring may sinasabi ito sa tendensiya ng mga Filipino na bumaling sa mga posibilidad sa halip na sa mga limitasyon, sa hinaharap sa halip na sa nakaraan.
}

(c) 2018 Noel L. Clemente

https://www.kritike.org/journal/issue 23/clemente december2018.pdf ISSN 1908-7330

(c) BY-NC-ND 
ang táong ito, at isa itong pagkakataong makilala siya at makipagkaibigan, o maaari ring pagkakataong isantabi siya at piliing hindi maging bahagi ng búhay ko.

Dito natin nauunawaan ang kasaysayan ng tao bilang isang pagkakataon: sabay na nagkataon lamang at wala nang magagawa ang tao, at isang pagkakataon na maaaring samantalahin ng tao. Manapa, naipapakita rin ito ng katagang pagtatakda. Kapag sinabi nating "naitakda na," may ibang entidad na nagpataw sa atin ng isang pangyayari, at wala na tayong magagawa rito. Ngunit ginagamit din natin ang salitang "takda" bilang isang gawain: na para bang takdang-aralin ng isang mag-aaral. Itinakda sa atin ang ating kasaysayan: muli, sabay na limitasyon at posibilidad. Katulad ng pagkakataon, nahuhúli rin ng pagtatakda ang dalawang mukha ng pagkasumasakasaysayan ng tao, ngunit mas naipapahiwatig nito ang kalayaan ng tao. Sapagkat limitado ang tao sa kaniyang kasaysayan, limitado rin ang kaniyang kalayaan. May mga "naitakda na" na hindi niya mababago. Sa kabilâng banda, anuman ang kaniyang limitasyon, naglalaman pa rin ito ng mga posibilidad, kayâ hindi rin masasabing radikal na determinado ang kaniyang kasaysayan.

Maaari rin nating iugnay ang pagiging pagkakataon ng kasaysayan sa salitang-ugat ng 'kasaysayan': saysay. Marahil, isa ito sa pinakapalasak na pagpapahayag ng pangunahing tanong sa ating búhay: Ano ba ang saysay ng ating búhay? Bakit ako umiiral? Ano ang papel ko sa mundong ito? Makatutulong sa paghahanap ng sagot sa tanong na ito ang pagkilala sa mga limitasyon at posibilidad ng ating pagkatao. Sa isang banda, ang saysay ko ay naitakda na ng aking nakaraan. Nagkataon na ganito ang kontekstong aking sinilangan, kayâ may hangganan ang mga maaari kong piliing landas. Gayunpaman, isa ring patuloy na takda ang paghahanap na ito ng saysay ng aking búhay; saanman ako naroroon sa kasalukuyan ay may pagkakataon akong pumili ng aking patutunguhan.

\section{Pilosopiya ng Tao bílang Pagpapahayag ng Tao po!}

Ang huli kong gustong pag-usapang kataga na nagpapalawig, hindi lang ng pamimilosopiya at ng pagiging tao, kundi ng mismong pamimilosopiya ukol sa tao, ay ang "tao po." Halaw ang pagtalakay na ito sa pagpapaliwanag ni Alejo ng pamagat ng kaniyang aklat. Sa Tao po! Tulóy!, inilalarawan at sinusuri ni Alejo ang loob, isang Filipinong kataga, konsepto, at katotohanan. Paliwanag niya, maaaring ihambing ang loob sa ibang mga obheto ng pilosopiya. Itinuring ng mga sinaunang Griyego ang katotohanan bilang aletheia, na nangangahulugang pag-alis mula sa pagkakakubli; samakatuwid kung gusto mong maunawaan ang aletheia, tingnan mo kung ano ang nagpapakita. Sentral naman kina Lao Tzu at Chuang Tzu ang Dao o 


\section{ILANG KATAGANG FILIPINO}

landas; kung gusto mong maunawaan ang Dao, bagtasin mo ang landas na ito. Sa gayon, kung gusto mong maunawaan ang loob ng tao, kailangan mong tumuloy sa loob. ${ }^{32}$ Ngunit, sa kaugaliang Filipino, hindi basta-basta ang pagpasok sa loob. Bílang paggalang, kailangan mo munang magpaálam, at karaniwang ipinapahayag itong pagpapaálam sa pagsambit ng "Tao po!" kasabay ng pagkatok sa pinto. At hinihintay niya muna ang tugon na "Tulóy" ng nása loob. Sa isang payak at pamilyar na palítang ito, marami tayong matututuhan ukol sa pakikiugnay ng mga tao.

Pagtuunan natin ng pansin ang mga katagang "tao po." May dalawa itong maaaring ipinahihiwatig. Una, isa itong pagpapahayag ng "May tao po rito!". Sa pamamagitan ng pagkatok sa pinto, nais mong ipaalam sa nása loob na may tao sa labas. Ikalawa, maaari rin itong pagtatanong o paghahanap ng tao sa loob, na para bang nagtatanong ng "May tao po ba riyan?" Ipinahihiwatig ng dalawang mukha nito ang karaniwang paghahati ng pagtuturo ng Pilosopiya ng Tao: sinisimulan ito sa mga tema ng pagtuklas sa sarili bílang tao (eksistensiyal), bago tumungo sa mga tema ng pagkilala sa ibang kapuwa tao (panlipunan). ${ }^{33}$ Ngunit sa pagbigkas ng "tao po," sa konteksto ng pagkatok bago pumasok sa loob, iminumungkahi ang sabay na pagpapahayag ng sarili at paghahanap ng kapuwa.

May matututuhan din tayo sa salitang kapuwa. Binabaybay rin ito bilang "kapwa," ngunit pinipili kong gamitin ang "kapuwa" dahil may iminumungkahi itong orihen ng salita. Maaaring tingnan ang "kapuwa" bilang pinaikling "ka-puwang," isang kahati sa puwang. ${ }^{34}$ Ang aking kapuwa ay ang tao na kabahagi ko sa puwang ng daigdig. Mapapansing hindi lamang pisikal o obhetibong puwang ang tinutukoy rito. Isa sa mga pinakamalalim na punto ng Pilosopiya ng Tao ay ang realisasyon na ano pa man ang ating angkan, kasarian, edad, o paniniwala, lahat tayo ay mga kapuwa tao, at iyon ang maaari nating pagsimulan ng pakikipag-ugnay.

Bilang pagpapatúloy ng pagtalakay sa "tao po," maaari nating idagdag ang pagsusuri ni Ricoeur sa Parabula ng Mabuting Samaritano. ${ }^{35}$

${ }^{32}$ Alejo, Tao pô! Tulóy!, 68.

33 Sa Pamantasang Ateneo de Manila, halimbawa, tinuturo ang Pilosopiya ng Tao sa dalawang magkasunod na kurso. Karamihan ng mga guro ay nagtutuon sa sarili sa PH101, at sa lipunan naman sa PH102.

${ }^{34}$ Kung tama ang aking pagkakatanda, sa isang klase ni Remmon E. Barbaza ko unang narinig ang ganitong mungkahi sa pahiwatig ng salitang "kapuwa." Sinubukan kong kumpirmahin ito sa diksiyonaryo, ngunit walang ganitong ipinapahayag na orihen. Gayunpaman, hindi imposibleng magkaugnay ang salitang ito sa "ka-puwang" na may malalim na sinasabi tungkol sa kahulugan nito. Nakikiusap ako sa ibang mananaliksik na mayroong masasabi sa ganitong lingguistikong pagsusuri, na mag-bahagi ng isa mang pagkumpirma o pagtuligsa, para rin maging tapat ang paggamit nitong salita sa kaniyang pinagmulan.

35 Paul Ricoeur, "Ang Kalipunan at ang Kapwa," in Pilosopiya ng Tao: Ilang Piniling Babasahin Tomo I, ed. by Antonette Palma-Angeles, Rofel G. Brion, et. al., trans. by Albert Alejo (Quezon City: Kagawaran ng Pilosopiya, Pamantasang Ateneo de Manila, 1988), 149-164,

(c) 2018 Noel L. Clemente

https://www.kritike.org/journal/issue 23/clemente december2018.pdf

ISSN 1908-7330

(cc) BY-NC-ND 
Aniya, ang kapuwa ay hindi sosyolohikal na kategoriya, kundi kilos ng sarili; ginagawa kong kapuwa ang sarili ko sa pakikipag-ugnay sa kaniya. Dito, nagsasabay ang danas ng 'ako' at ng 'kapuwa'; nagiging kapuwa ako sa pamamagitan ng aking kilos. Samakatuwid, hindi hiwalay ang ako at ang kapuwa. Hindi ko mauunawaan ang ako nang hiwalay sa kapuwa, at hindi ko mauunawaan ang kapuwa nang hiwalay sa akin. Ito ang nagaganap sa pamimilosopiya ng tao. Kumakatok ako sa pinto ng aking kapuwa, binibigkas ang "Tao po!" bilang pagpapakilala ng sarili ko bilang tao, samantalang hinahanap ang kapuwa ko na alam kong katulad kong tao.

Tulad ng nabanggit sa ikalawang seksiyon, ang pamimilosopiya ay mas maihahanay sa pagmumuning sekundarya, kung saan isinasangkot ko ang sarili ko. Kaakibat ng pagsangkot ng aking sarili ang pagtataka ukol sa kapuwa. Samakatwid, nakikilala ko ang kapuwa hindi sa paraan ng pagsisiyasat sa kaniya na para bang isang specimen sa isang laboratoryo, kundi sa aking mismong pakikihalubilo sa kaniya. Kaakibat nito, lalo ko ring nakikilala ang sarili ko sa pakikiugnay sa iba-sabay na paglantad ng sarili, "May tao po rito!", sa paghahanap ng makauugnay na kapuwa, "May tao po ba riyan?"

\section{Pangwakas}

Taliwas sa palagay ni Quito, mayamang-mayaman ang ating wika sa mga salita na hitik sa pilosopikong kahulugan. Marami táyong matututuhan sa pagsisiyasat ng ating mga kataga; ang aking mga iminungkahi sa mga nakaraang bahagi ay ilan lamang halimbawang panimula. Hindi man maiiwasan na ihambing ang mga nabanggit ko sa mga Europeong konsepto na marahil mas kilalá na sa akademya, igigiit kong iba pa rin ang danas ng ating mga salita sa kanila.

Bilang pangwakas, nais kong magbigay pa ng dalawa pang mga katagang Filipino na masasabi ring bukál ng pilosopikong diskurso. Tulad ng mga tinalakay kong kataga sa mga naunang seksiyon, masasabing nakapaloob sa mga katagang ito ang mga nasabi na ng mga pilosopong Europeo, na nagpapahiwatig na buháy sa ating kultura ang danas ng kanilang mga nasabi. Ipaliliwanag ko lang nang bahagya ang kaugnayan ng mga ibibigay kong salita sa mga pilosopikong ideya, ngunit iiwan kong bukás ang diskusyon sa mga katagang ito.

Isang karaniwang paksa sa Pilosopiya ng Tao ang mga pananaw ni Karl Marx ukol sa alyenadong paggawa. Ginagamit nating salita sa trabaho ang hanapbuhay, na nagpapahiwatig ng sinasabi niyang ikatlong anyo ng 


\section{ILANG KATAGANG FILIPINO}

alyenasyon, ang alyenasyon sa pag-iral na pantao. ${ }^{36}$ Ayon kay Marx, dulot ng alyenasyon ng manggagawa sa produkto at sa mismong akto ng paggawa, ibinubuhos ng manggagawa ang kaniyang sarili sa produktong hindi nagiging kaniya. Dahil dito, nagiging banyaga sa kaniya ang pag-iral na bukod tanging para sa mga tao lamang: ang kakayahang gumawa nang lagpas sa kinakailangan. Nakapagtatrabaho rin naman, halimbawa, ang mga langgam sa paggawa nila ng punso, mga bubuyog sa paglikha nila ng bahaypukyutan, o mga gagamba sa paghabi nila ng sapot, ngunit ang mga ito ay tawag ng pangangailangan nilang mabuhay. Tao lamang, para kay Marx, ang may kakayahang lumikha ng mga bagay na hindi naman niya kinakailangan upang mabuhay, tulad ng smartphone, tula, awit, puntod, atbp. Subalit sa paggawa ng manggagawa ng produktong hindi mapapasakaniya, napapako ang kaniyang paggawa sa pangangailangan, at maihahambing na lang siya sa mga langgam, bubuyog, at gagamba. Samakatuwid, kung ang trabaho ay hamak na paghahanap-ng-búhay, sa literal na kahulugan na ginagawa niya ito para lamang mabuhay, nakararanas siya ng alyenadong paggawa.

Maaari ring suriin ang katagang karapatan. Ang salitang ugat nito ay 'dapat.' Ang karapatan ay ang napagkasunduang mga "dapat" ng isang pamayanan. Kung nais ng mga mamamayan na mamuhay nang matiwasay, kailangan nilang igalang ang karapatan ng isa't isa, at kaugnay nito, kailangan nilang sundin ang mga kaugnay na patakaran. (Nakatutuwang punahin na anagrama ng "karapatan" ang "patakaran.") Ngunit parating may konteksto ang mga karapatan; nagkakaroon ang isang entidad ng karapatan bilang bahagi ng isang pangkat. Ang mga karapatang pantao ay karapatan natin bilang mga tao (kaya nga may Universal Declaration of Human Rights ang United Nations; unibersal ang mga karapatang ito para sa lahat ng tao); ang mga karapatang sibil ay karapatan ng isang mamamayan bilang napapasailalim sa isang pamahalaan; ang mga karapatang pambabae ay karapatan na natamo sa mismong pagiging babae. Kaugnay ng pagiging bahagi ng isang pangkat, mayroong mga nararapat (at di-nararapat) para sa mga taong ito. Mula rito, maaari pang suriin ang katagang "karapatan" bilang pundasyon ng isang pilosopiyang panlipunan nating mga Filipino.

Umaasa akong ang pagbabahaging ito ng mga ideya hinggil sa mga salitang Filipino na maituturing na bukal ng diwang pilosopiko ay maging isang munting ambag sa pagpapatuloy ng tradisyon ni Ferriols ng pagtuturo ng pilosopiya at pamimilosopiya sa wikang Filipino. Hinihimok ko ang aking mga mambabasa na ipagpatuloy ang ganitong pagpapahalaga at paglilinang sa ating wika sa larangan ng pamimilosopiya.

36 Ang "pag-iral na pantao" ay salin ng may-akda sa "species-being." Karl Marx, "Alienated Labor," in Karl Marx: Selected Writings, 2nd ed., ed. by David McLellan (Oxford: Oxford University Press, 2000), 89-91.

(C) 2018 Noel L. Clemente

https://www.kritike.org/journal/issue 23/clemente december2018.pdf ISSN 1908-7330

(c) $)$ BY-NC-ND 


\section{Pasasalamat at Pagkilala}

Nais kong pasalamatan sina Precious De Joya at Albert Alejo na humimok sa aking isulat ang papel na ito, pati na rin ang mga tagapagbasáng napili ng Kritike para sa kanilang mahahalagang puna sa unang burador nitong manuskrito. Nais ko ring kilalanin ang mga ambag at mungkahi nina Danna Aduna, Jayne Tinhay Arriola, at Donald Jay Bertulfo.

Department of Philosophy, Ateneo de Manila University, Philippines

\section{References}

Alejo, Albert E., Tao pô! Tulóy! Isang Landas ng Pag-unawa sa Loob ng Tao (Quezon City: Ateneo de Manila University Office of Research and Publications, 1990).

Aristotle, Metaphysics, trans. by W.D. Ross, in The Basic Works of Aristotle, ed. by Richard McKeon. (New York: Random House, 1941).

Calano, Mark Joseph, "Ang Pilosopiya at si Roque J. Ferriols, SJ," Perspectives in the Arts and the Humanities 5:1 (2015)

Cortez, Franz, Giuseppe F., "The Linguistic Turn as a Political Act: Another Look at the Thoughts of Roque Ferriols," Kritike: An Online Journal of Philosophy, 8:1 (June 2014).

Ferriols, Roque J., “A Memoir of Six Years,” Philippine Studies 22 (1974).

Pambungad sa Metapisika (Quezon City: Ateneo de Manila University Press, 2014)

Lagliva, Albert M., "Ang Paraan at mga Elemento ng Pagtuturo ni Roque J. Ferriols, SJ, tungo sa Matinong Pag-Uunawa," Perspectives in the Arts and Humanities Asia 5:1 (2015).

Marcel, Gabriel, “Ang Bulangang 'Heto Ako!',” trans. by Nonna J. Peña, in Magpakatao: Ilang Babasahing Pilosopiko, 2nd ed., ed. by Roque J. Ferriols (Quezon City: Office of Research and Publications, Ateneo de Manila University, 1999).

Reflection and Mystery, vol. 1 of The Mystery of Being, trans. by G.S. Fraser (London The Harvill Press, 1951).

Marx, Karl, "Alienated Labor," in Karl Marx: Selected Writings, 2nd ed., ed. by David McLellan (Oxford: Oxford University Press, 2000)

Mercado, Leonardo N., Elements of Filipino Philosophy (Tacloban City: Divine Word University Publications, 1993).

Oris, Glenda C., "Ferriols on Ferriols and More," Perspectives in the Arts and Humanities Asia 5:1 (2015).

(C) 2018 Noel L. Clemente

https://www.kritike.org/journal/issue 23/clemente december2018.pdf

ISSN 1908-7330 


\section{ILANG KATAGANG FILIPINO}

Que, Nemesio S. and Agustin Martin G. Rodriguez, eds., Introduction to Pagdiriwang sa Meron: A Festival of Thinking Celebrating Fr. Roque J. Ferriols, S.J. (Quezon City: Ateneo de Manila University, 1997).

Quito, Emerita S., "Ang Kayamanan ng Wikang Filipino," in A Life of Philosophy: Festschrift in Honor of Emerita S. Quito (Manila: De La Salle University Press, 1990).

Lectures on Comparative Philosophy (Manila: De La Salle University Press, 1979).

Reyes, Ramon, "Man and Historical Action," in Philosophy of Man: Selected Readings, 3rd ed., ed. by Manuel B. Dy, Jr. (Quezon City: Katha Publishing Co., Inc., 2012).

Ricoeur, Paul, "Ang Kalipunan at ang Kapwa," in Pilosopiya ng Tao: Ilang Piniling Babasahin Tomo I, ed. by Antonette Palma-Angeles, Rofel G. Brion, et. al., trans. by Albert Alejo (Quezon City: Kagawaran ng Pilosopiya, Pamantasang Ateneo de Manila, 1988).

Strebel, Wilhelm P.J., "Pitong Sulyap sa Pilosopiya ng Wika ni Ferriols," Kritike: An Online Journal of Philosophy, 12:1 (June 2018).

Tolentino, Roy Allan B., "Biyaya ang Meron, Biyaya ng Meron: Ang Pag-iisip nina Ferriols, Marion, at San Agustin," Perspectives in the Arts and Humanities Asia 5:1 (2015).

Wittgenstein, Ludwig, Philosophical Investigations, rev. 4th ed., ed. by P.M.S. Hacker and Joachim Schulte, trans. by G.E.M. Anscombe, P.M.S. Hacker, and Joachim Schulte (Malden: Wiley-Blackwell, 2009). 